\title{
Lower Mass Bound on the $W^{\prime}$ Mass via Neutrinoless Double Beta Decay in a 3-3-1 Model
}

\author{
A. C. O. Santos $\mathbb{D}^{1,2}$ and P. Vasconcelos $\mathbb{D}^{1}$ \\ ${ }^{1}$ Departamento de Física, Universidade Federal da Paraíba, Caixa Postal 5008, 58051-970 João Pessoa, PB, Brazil \\ ${ }^{2}$ Centre for Cosmology, Particle Physics and Phenomenology (CP3), Université Catholique de Louvain, \\ 1348 Louvain-la-Neuve, Belgium
}

Correspondence should be addressed to A. C. O. Santos; antonio_santos@fisica.ufpb.br

Received 10 November 2017; Revised 19 February 2018; Accepted 3 April 2018; Published 23 May 2018

Academic Editor: Jose W. F. Valle

Copyright (C) 2018 A. C. O. Santos and P. Vasconcelos. This is an open access article distributed under the Creative Commons Attribution License, which permits unrestricted use, distribution, and reproduction in any medium, provided the original work is properly cited. The publication of this article was funded by SCOAP ${ }^{3}$.

The discovery of neutrino masses has raised the importance of studies in the context of neutrinoless double beta decay $(0 \nu \beta \beta)$, which constitutes a landmark for lepton number violation (LNV). The standard interpretation is that the light massive neutrinos that we observed oscillating in terrestrial experiments mediate double beta decay. In the minimal 3-3-1 model (3-3-1M), object of our study, there is an additional contribution that stems from the mixing between a new charged vector boson, $W^{\prime}$, and the Standard Model $W$ boson. Even after setting this mixing to be very small, we show that tight constraints arise from the nonobservation of $0 v \beta \beta$. Indeed, we derive bounds on the mass of the $W^{\prime}$ gauge boson that might exceed those from collider probes and most importantly push the scale of symmetry breaking beyond its validity, leading to an exclusion bound for the minimal 3-3-1 model.

\section{Introduction}

The Standard Model (SM) has thrived after a multitude of precision tests at low and high energy scales [1]. However, the existence of neutrino masses is one of the main motivations for physics beyond the SM [2]. If neutrinos are majorana particles, neutrinoless double beta decay $(0 \nu \beta \beta)$ should occur. Double beta decay is the transition of a nucleus with mass and atomic number $A$ and $Z$ to a nucleus with $A$ and $Z+2$, accompanied by the emission of two electrons only. Its possible discovery will represent an irrefutable proof of lepton number violation, LNV. The standard diagram that leads to such lepton number violation process is exhibited in Figure 1.

At present, a lot of experiments using different isotopes and techniques are operating or under development in the search for neutrinoless double beta decay (see [3]). In this work we investigate the implications of the nonobservation of neutrinoless double beta decay in the context of 3-3-1 models [4-9]. 3-3-1 models are plausible extensions of the SM where fermions are placed in the fundamental or adjoint representation of $S U(3)$. Generally these models while being consistent with colliders data $[10,11]$ nicely explain why we have three fermion generations [5, 12, 13], accommodate neutrino masses [14-16], and may feature several dark matter candidates $[17,18]$ to the dark matter problem. Moreover, they could also address low energy anomalies $[19,20]$ and might feature interesting astrophysical [21,22] and collider phenomenology via the presence of exotic gauge bosons which are popularly used in many different contexts [23, 24]. See [25-31] for many other exciting phenomenological studies.

In this work, we focus our attention on the minimal 33-1 model (3-3-1M), one of the possible versions of 3-3-1 models, in which the electric operator takes the form $Q=$ $T_{3}+\beta T_{8}+X$ considering $T_{i}$ as $\lambda^{a} / 2$ (Gell-Mann matrices with $a=1, \ldots, 8), \beta=-\sqrt{3}$ for $S U(3)_{L}$ (see explanation below), and $X$ as $U(1)_{X}$ charge, where no new leptons are evoked (right-handed neutrinos as well), thus featuring a minimal fermion content. Since we are dealing with an extended gauge group, there will be additional gauge bosons, one of them being singly charged, $W^{\prime}$, due to the chosen charge operator parameter. This new gauge boson might induce the 


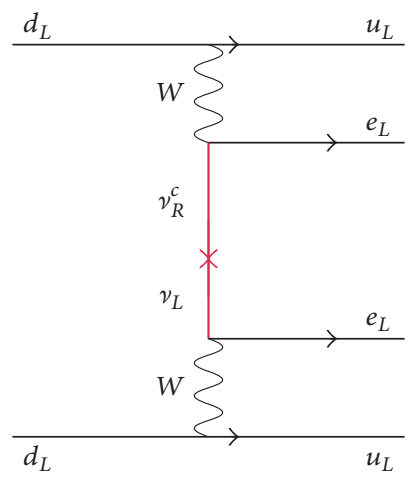

Figure 1: Canonical $0 \nu \beta \beta$ due to light massive neutrinos.

neutrinoless double beta decay if it mixes with the $W$ boson. In order to successfully explain neutrino masses in this model without the use of nonrenormalizable operators [14], a scalar sextet should be introduced $[32,33]$. This scalar sextet embeds the scalar triplet model, often used in the context of neutrino masses [34-37]. The first component of the scalar sextet is a neutral field which is often assumed to yield a null vacuum expectation value (vev). In this work we make assumption otherwise. If such scalar acquires a nonvanishing vev then $W^{\prime}$ mixes with the SM $W$ boson, giving rise to new contributions to the neutrinoless double beta decay. This scenario has been investigated before in the context of majoron in [38-41]. Here we revisit the implications in a more general setting in perspective with other existing colliders constraints. We will ignore contributions arising from the scalar fields because they yield less restrictive bounds [39] and because a bound on the $W^{\prime}$ mass is already implied into a lower mass bound on the entire particle spectrum of model, since the $W^{\prime}$ mass is directly connected to the scale of symmetry breaking of the model. It is worth mentioning that in this work our parameter space provides a very small $Z-Z^{\prime}$ mixing and does not bring relevant constraints coming from STU parameters [41, 42].

We will show that if $W^{\prime}$ does feature a mixing with the SM $W$ boson then limits stemming from neutrinoless double beta decay supersede LHC probes, highlighting the importance of multiple new physics searches probes.

The paper is organized as follows: In Section 2 we describe the model and explain how the $W^{\prime}-W$ mass mixing can be generated. In Section 3 we derive the contributions to neutrinoless double beta decay. In Section 4 we draw our conclusions.

\section{The Model}

In the minimal 3-3-1 model leptons are arranged as

$$
L^{\ell}=\left(\begin{array}{c}
v_{\ell} \\
\ell \\
\ell^{c}
\end{array}\right)_{L} \sim(3,0),
$$

where $\sim$ represents the way the triplet transforms under $S U(3)_{L} \otimes U(1)_{N}$, with $\ell=e, \mu, \tau$, representing the three known generations. Notice that the third component of the lepton triplet is the right-handed lepton. The quarks are placed as follows:

$$
\begin{aligned}
Q_{1 L} & =\left(\begin{array}{c}
u_{1} \\
d_{1} \\
J_{1}
\end{array}\right)_{L} \sim\left(3, \frac{2}{3}\right), \\
Q_{i L} & =\left(\begin{array}{c}
d_{i} \\
-u_{i} \\
J_{i}
\end{array}\right)_{L} \sim\left(\overline{3},-\frac{1}{3}\right), \quad i=2,3 .
\end{aligned}
$$

Here $u_{a L}$ and $d_{a L}(a=1,2,3)$ correspond, respectively, to flavors $u, c, t$ and $d, s, b$ of the Standard Model (SM) quarks. The first generation transforms as a triplet, while the second and third generations transform as antitriplets under $S U(3)_{L}$. In addition to the left-handed field we have right-handed quarks as singlets under $S U(3)_{L}: u_{a R} \sim(1,2 / 3), d_{a R} \sim$ $(1,-1 / 3), J_{1 R} \sim(1,5 / 3), J_{i R} \sim(1,-4 / 3)$, with $a=1,2,3$ and $i=2,3$. $J_{1}$ and $J_{i}$ are exotic quarks predicted by the model with electric charges of $5 / 3$ and $-4 / 3$, respectively. These exotic quarks are also known as leptoquarks in the literature $[43,44]$.

The mass generation mechanism relies firstly on the presence of three scalar triplets; namely,

$$
\begin{aligned}
& \eta=\left(\begin{array}{l}
\eta^{0} \\
\eta_{1}^{-} \\
\eta_{2}^{+}
\end{array}\right), \\
& \rho=\left(\begin{array}{c}
\rho^{+} \\
\rho^{0} \\
\rho^{++}
\end{array}\right), \\
& \chi=\left(\begin{array}{c}
\chi^{-} \\
\chi^{--} \\
\chi^{0}
\end{array}\right),
\end{aligned}
$$

with them transforming as $(3,0),(3,1)$, and $(3,-1)$ under $S U(3)_{L} \otimes U(1)_{N}$, respectively. These scalar triplets couple with the fermions fields through the following Yukawa Lagrangian:

$$
\begin{aligned}
-\mathscr{L}_{Y}= & \frac{1}{2} \sum_{\ell \ell^{\prime}} G^{\ell \ell^{\prime}} \epsilon^{l m n}\left[\overline{\left(L_{l}^{\ell}\right)^{c}} L_{m}^{\ell^{\prime}} \eta_{n}\right]+G_{1 a}^{u} \bar{Q}_{1 L} \eta u_{a R} \\
& +G_{1 a}^{d} \bar{Q}_{1 L} \rho d_{a R}+G_{11}^{J 1} \bar{Q}_{1 L} \chi J_{1 R}+G_{2 a}^{s} \bar{Q}_{2 L} \eta^{*} d_{a R} \\
& +G_{2 a}^{c} \bar{Q}_{2 L} \rho^{*} u_{a R}+G_{2 i}^{J 2} \bar{Q}_{2 L} \chi^{*} J_{i R} \\
& +G_{3 a}^{b} \bar{Q}_{3 L} \eta^{*} d_{a R}+G_{3 a}^{t} \bar{Q}_{3 L} \rho^{*} u_{a R} \\
& +G_{3 i}^{J 3} \bar{Q}_{3 L} \chi^{*} J_{i R}+\text { h.c., }
\end{aligned}
$$

with $\ell, \ell^{\prime}=e, \mu, \tau$. Equation (4) is sufficient to generate masses to charged leptons and quarks but neutrinos remain 
massless. In order to obtain nonzero neutrino masses, a scalar sextet should be introduced:

$$
S=\left(\begin{array}{ccc}
\sigma_{1}^{0} & \frac{h_{2}^{-}}{\sqrt{2}} & \frac{h_{1}^{+}}{\sqrt{2}} \\
\frac{h_{2}^{-}}{\sqrt{2}} & H_{1}^{--} & \frac{\sigma_{2}^{0}}{\sqrt{2}} \\
\frac{h_{1}^{+}}{\sqrt{2}} & \frac{\sigma_{2}^{0}}{\sqrt{2}} & H_{2}^{++}
\end{array}\right) \sim(\mathbf{6}, \mathbf{0})
$$
term,

The presence of such scalar sextet gives rise to the Yukawa

$$
\mathscr{L}_{Y}^{S}=-\frac{1}{2} \sum_{\ell \ell^{\prime}} G \overline{\left(L^{\ell}\right)^{c}} S^{*} L^{\ell^{\prime}}
$$

with $L^{c}=C \bar{L}^{T}, C$ being the charge conjugate matrix.

Expanding (6) we have explicitly

$$
\begin{aligned}
& \mathscr{L}_{Y}^{S} \supset-\frac{1}{2} \sum_{\ell \ell^{\prime}} G_{\ell \ell^{\prime}}\left[\overline{\left(v_{\ell L}\right)^{c}} v_{\ell^{\prime} L} \sigma_{1}^{0}+\overline{\left(\ell_{L}\right)^{c}} \ell_{L}^{\prime} H_{1}^{++}\right. \\
& +\overline{\ell_{R}}\left(\ell^{\prime c}\right)_{L} H_{2}^{--}+\left(\overline{\left(\ell_{L}\right)^{c}} v_{\ell^{\prime} L}+\overline{\left(v_{\ell L}\right)^{c}} \ell_{L}^{\prime}\right) \frac{h_{2}^{+}}{\sqrt{2}} \\
& +\left(\overline{\ell_{R}} v_{\ell^{\prime} L}+\overline{\left(v_{\ell L}\right)^{c}}\left(\ell^{\prime c}\right)_{L}\right) \frac{h_{1}^{-}}{\sqrt{2}} \\
& \left.+\left(\overline{\ell_{R}} \ell_{L}^{\prime}+\overline{\left(\ell_{L}\right)^{c}}\left(\ell_{R}^{\prime}\right)^{c}\right) \frac{\sigma_{2}^{0}}{\sqrt{2}}\right]+ \text { h.c. }
\end{aligned}
$$

Notice that a new contribution to the charged lepton masses arises if $v_{\sigma_{2}} \neq 0$. Moreover, neutrinos remain massless unless $\left\langle\sigma_{1}^{0}\right\rangle \neq 0$. Typically, this scalar sextet is absent in 3-3-1 studies, and consequently neutrino masses are not addressed. In this work, we discuss a more general setting, where the scalar sextet is present and $v_{\sigma_{1,2}} \neq 0$. In this setup, the spontaneous symmetry breaking occurs as follows: firstly, $\chi^{0}$ develops vev $\left(v_{\chi}\right)$, breaking $S U(3)_{L} \otimes U(1)_{N}$ into $S U(2)_{L} \otimes U(1)_{Y}$. Later $\rho^{0}$ and $\eta^{0}$ acquire a nonzero vev with $v_{\rho} \sim v_{\eta}$, breaking $S U(2) \otimes U(1)_{Y}$ into $U(1)_{\mathrm{QED}}$, that is, the electromagnetism gauge group.

This symmetry breaking pattern generates mass to all SM fermions and gauge bosons. We highlight that, due to the enlarged gauge group, 3-3-1 models feature five new gauge bosons. In the minimal 3-3-1 model they are identified as $W^{\prime \pm}, U^{ \pm \pm}$, and $Z^{\prime}$. An important result of the spontaneous symmetry breaking is the rising of a mass mixing between the $W$ and $W^{\prime}$ bosons that yields the mass mixing matrix:

$$
\left(\begin{array}{ll}
W_{\mu}^{+} & W_{\mu}^{\prime+}
\end{array}\right)\left(\begin{array}{cc}
M_{W}^{2} & M_{W W^{\prime}}^{2} \\
M_{W W^{\prime}}^{2} & M_{W^{\prime}}^{2}
\end{array}\right)\left(\begin{array}{c}
W^{\mu-} \\
W^{\prime \mu-}
\end{array}\right),
$$

where

$$
M_{W W^{\prime}}^{2}=\frac{g^{2}}{2}\left(2 v_{\sigma_{1}} v_{\sigma_{2}}\right)
$$

$g=e / s_{W}$, and

$$
\begin{aligned}
& M_{W}^{2}=\frac{g^{2}}{2}\left(v_{\eta}^{2}+v_{\rho}^{2}+v_{\sigma_{2}}^{2}+v_{\sigma_{1}}^{2}\right), \\
& M_{W^{\prime}}^{2}=\frac{g^{2}}{2}\left(v_{\eta}^{2}+v_{\chi}^{2}+v_{\sigma_{2}}^{2}+v_{\sigma_{1}}^{2}\right) .
\end{aligned}
$$

If we take $v_{\sigma_{1,2}}=0$, then $M_{W W^{\prime}}^{2}=0$, and consequently $v_{\eta}^{2}+v_{\rho}^{2}=v^{2}$, where $v=175 \mathrm{GeV}$. In this way, the $W$ mass is correctly obtained. After diagonalization we find two mass eigenstates $W_{1}^{+}$and $W_{2}^{+}$with

$$
\begin{aligned}
& M_{W_{1,2}}=\frac{1}{2}\left[\left(M_{W}^{2}+M_{W^{\prime}}^{2}\right)\right. \\
& \left.\quad \pm\left(\left(M_{W}^{2}-M_{W^{\prime}}^{2}\right)^{2}+4 M_{W W^{\prime}}^{4}\right)^{1 / 2}\right],
\end{aligned}
$$

which are related to the mass eigenvectors via

$$
\left(\begin{array}{c}
W^{+} \\
W^{\prime+}
\end{array}\right)=\left(\begin{array}{cc}
c_{\theta} & -s_{\theta} \\
s_{\theta} & c_{\theta}
\end{array}\right)\left(\begin{array}{c}
W_{1}^{+} \\
W_{2}^{+}
\end{array}\right),
$$

with

$$
\tan 2 \theta=\frac{-2 M_{W W^{\prime}}^{2}}{\left(M_{W}^{2}-M_{W^{\prime}}^{2}\right)}
$$

Considering the limit $M_{W W^{\prime}}^{2} \rightarrow 0$, we get $M_{W_{1}}=M_{W}$ and $M_{W_{2}}=M_{W^{\prime}}$. The charged currents associated with these gauge bosons are

$$
\begin{aligned}
& \mathscr{L}_{\mathrm{cc}} \supset \frac{g}{\sqrt{2}}\left[\bar{u}_{L} \gamma^{\mu} V_{C K M} d_{L}-\bar{\nu}_{L} \gamma^{\mu}\left(U_{v} V_{\ell}\right) l_{L}\right] W_{\mu}^{+} \\
&+\frac{g}{\sqrt{2}}\left[\bar{l}^{c} \gamma^{\mu}\left(V_{l} U_{\nu}^{\dagger}\right) \nu_{L}\right] W_{\mu}^{\prime+}+\text { h.c. },
\end{aligned}
$$

where $V_{l}$ and $U_{v}$ are the mixing matrices for the charged leptons and neutrinos, with $\nu_{L}=\left(v_{e L}, v_{\mu L}, v_{\tau L}\right)$ and $l_{L}=$ $\left(e_{L}, \mu_{L}, \tau_{L}\right)$. There is also a term involving the doubly charged gauge boson, but it is not relevant for our reasoning [40].

We have gathered all ingredients to now discuss the neutrinoless double beta decay in the minimal 3-3-1 model.

\section{The Neutrinoless Double Beta Decay}

Neutrinoless double beta decay is a landmark process in particle physics. It is defined as the transition of a nucleus into another nucleus with an atomic number larger by two units and the emission of two electrons only:

$$
(A, Z) \longrightarrow(A, Z+2)+2 e^{-} .
$$

Since there are no leptons in the initial state, but two in the final state, the observation of neutrinoless double beta decay constitutes an irrefutable proof that lepton number is violated by nature. In the past decades, there has been a substantial improvement on the bound over the half-life of 


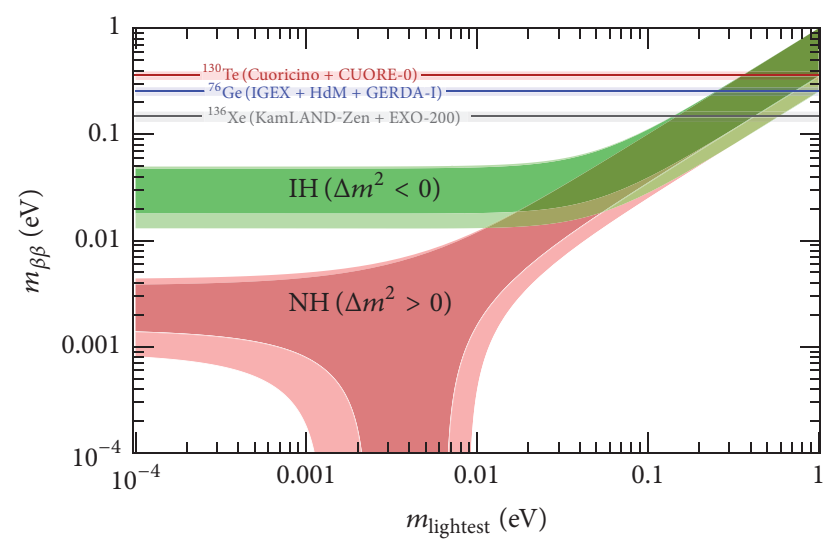

FIGURE 2: Updated prediction on $m_{\beta \beta}$ from neutrino oscillations as a function of the lightest neutrino mass according to the current neutrino data. In the figure, both normal and inverted hierarchy schemes for neutrino masses are displayed. Figure taken from [45]. In the figure is also visible the current limit from KamLAND-Zen.

the neutrinoless double beta decay [45]. These bounds can be later translated into upper limits on the effective majorana mass defined as

$$
m_{\beta \beta}=\left|\sum_{i=e, \mu, \tau} U_{e i}^{2} m_{v_{i}}\right| .
$$

The effective majorana mass term grows inversely with the half-life. A stronger lower bound on the half-life of the neutrinoless double beta decay implies a stronger bound on the effective majorana mass term (see Figure 2). The strongest bound today comes from KamLAND-Zen [46], $t_{1 / 2}>2.6 \times$ $10^{25} \mathrm{yrs}$, implying that

$$
m_{\beta \beta}^{\text {current }}<0.15 \mathrm{eV} .
$$

In the foreseeable future, CUORE is expected to achieve ${ }^{130} \mathrm{Te}>9.5 \times 10^{25}$ yrs [45] which translates into

$$
m_{\beta \beta}^{\text {CUORE }}<0.073 \mathrm{eV} \text {. }
$$

A projected limit further into the future with nEXO of $t_{1 / 2}>10^{27}$ yrs for ${ }^{136} \mathrm{Xe}$ is expected, which then would yield

$$
m_{\beta \beta}^{\mathrm{nEXO}}<0.01 \mathrm{eV} .
$$

Now from the current and projected experimental sensitivity we will approach the theoretical aspects of this observable. That said, the amplitude in Figure 1 is proportional to

$$
A_{1} \propto \frac{g^{4} m_{\beta \beta}}{M_{W}^{4}\left\langle p^{2}\right\rangle} c_{\theta}^{4}=\frac{32 G_{F}^{2} m_{\beta \beta}}{\left\langle p^{2}\right\rangle} c_{\theta}^{4},
$$

where $\left\langle p^{2}\right\rangle$ is the average of the four-momentum transfer squared, which is approximately $(100 \mathrm{MeV})^{2}$.

Moreover, one should observe that the amplitude for the diagram in Figure 3 is proportional to

$$
A_{2} \propto 32 G_{F}^{2}\left(\frac{M_{W}}{M_{W^{\prime}}}\right)^{2} \frac{c_{\theta}^{3} s_{\theta}}{\sqrt{\left\langle p^{2}\right\rangle}} .
$$

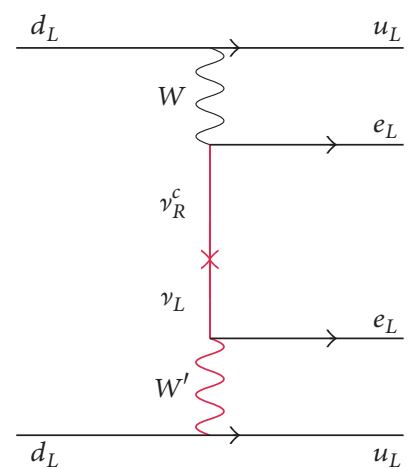

FIgURE 3: Novel 331 contribution for double beta decay due to light massive neutrinos.

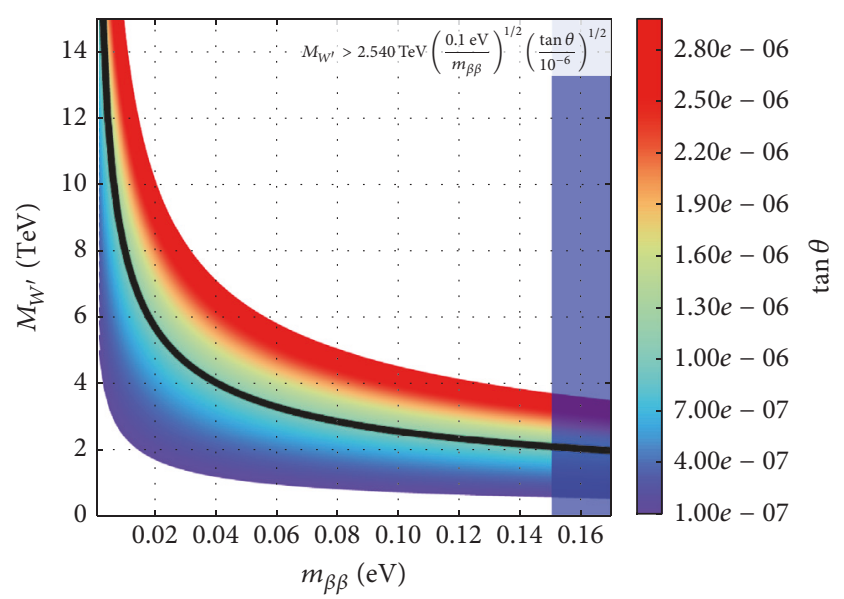

FIGURE 4: Temperature plot of the lower mass bound on the $W^{\prime}$ mass as function of $m_{\beta \beta}$ and $\tan \theta$. The black curve is drawn simply to guide the eye and check that, indeed for $\tan \theta \sim 10^{-6}$ and $m_{\beta \beta} \sim$ $0.1 \mathrm{eV}$, we find the lower mass bound $M_{W^{\prime}} \gtrsim 2.5 \mathrm{TeV}$.

Bearing in mind that $\theta$ should be small in order not to alter the SM $W$ properties, then $A_{2} / A_{1}<1$. This results in

$$
\frac{A_{2}}{A_{1}}=\left(\frac{M_{W}}{M_{W^{\prime}}}\right)^{2} \frac{\sqrt{\left\langle p^{2}\right\rangle}}{m_{\beta \beta}} \tan \theta<1 .
$$

Therefore we get the lower mass bound:

$$
M_{W^{\prime}}>2.540 \mathrm{TeV}\left(\frac{0.1 \mathrm{eV}}{m_{\beta \beta}}\right)^{1 / 2} \times\left(\frac{\tan \theta}{10^{-6}}\right)^{1 / 2} .
$$

In Figure 4 we exhibited this lower mass bound on the $W^{\prime}$ mass. It is quite visible that even for small $\tan \theta$ the bound on $W^{\prime}$ is rather strong. It is important to make it clear that the 3-3-1M models give us other contributions beyond the one depicted in Figure 3, as pointed out by [47], but they are of much smaller intensity and therefore do not give new better results.

However, equation (23) is not very useful to us because the $W^{\prime}$ mass also appears in $\tan \theta$. Therefore, these quantities are not independent; they are strongly correlated. Bearing in 


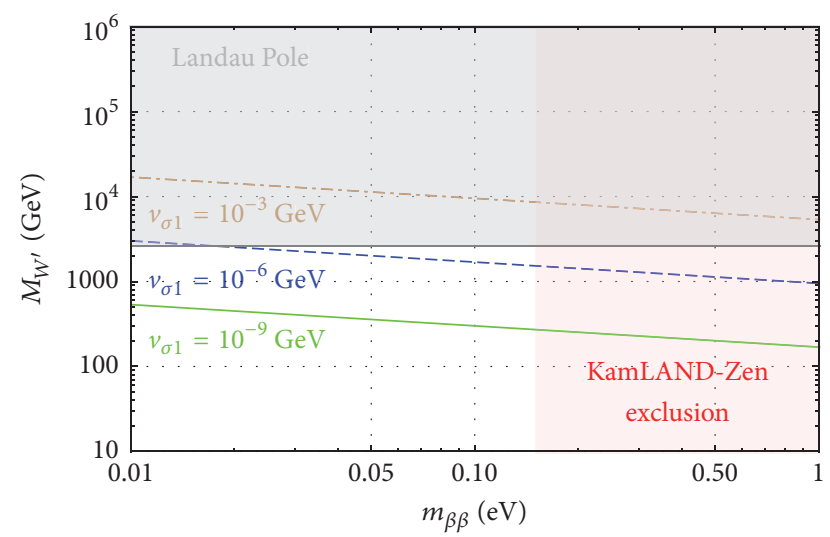

Figure 5: Lower mass bound on the $W^{\prime}$ mass from the nonobservation of the neutrinoless double beta decay with different choices for the vev of scalar field $\sigma_{1}$ which controls the parameter $M_{W W^{\prime}}^{2}$ in (24). The gray solid area denotes Landau pole upper limit [12, 50] and light red denotes the KamLAND-Zen exclusion [46].

mind that we are working in the regimen where $\theta \ll 1$, then $\tan \theta \sim \theta$ and we can solve for $M_{W^{\prime}}$ to find

$$
M_{W^{\prime}}>50.4 \mathrm{TeV}\left(\frac{0.1 \mathrm{eV}}{m_{\beta \beta}}\right)^{1 / 4}\left(M_{W W^{\prime}}^{2}\right)^{1 / 4}
$$

This equation can give rise to more robust bounds on the $W^{\prime}$ mass since the parameters that go into it are the majorana effective mass $m_{\beta \beta}$ and $M_{W W^{\prime}}^{2}$ term, which depends on vevs of the scalar fields $\sigma_{1}$ and $\sigma_{2}$. Since the Standard Model $W$ mass does not come mostly from vevs of these scalar fields as long as they are much smaller than $v_{\mathrm{SM}}$, then our choices for $v_{\sigma_{1}}$ and $v_{\sigma_{2}}$ are in principle completely arbitrary.

To have a clear vision of what (24) represents, we show the lower mass bounds on the $W^{\prime}$ mass in Figure 5. There we select $v_{\sigma 2}=3 \mathrm{GeV}$; larger values are ruled out by the $\rho$-parameter $[39,48]$ and vary $v_{\sigma 1}$. Notice that, depending on the value adopted for $v_{\sigma 1}$, neutrinoless double beta decay might yield very strong constraints on the $W^{\prime}$ mass. In particular for $m_{\beta \beta} \sim 0.1 \mathrm{eV}$ we get a lower mass bound on the $W^{\prime}$ mass that varies from $300 \mathrm{GeV}$ to about $9.5 \mathrm{TeV}$. The latter is achieved if $v_{\sigma 1}=10^{-3} \mathrm{GeV}$.

One shortcoming of our lower mass bound on the $W^{\prime}$ mass is its dependence on the choice for vevs. Anyways, these constitute an independent bound on the $W^{\prime}$ mass which can be much stronger than the limit on the $W^{\prime}$ arising from colliders searches. Notice that our $W^{\prime}$ does not couple only to ordinary quarks; therefore it cannot be produced resonantly at the LHC via s-channel processes. Nevertheless, one can use LHC limits on the $Z^{\prime}$ mass to convert into a lower mass limit on the $W^{\prime}$ mass, since both masses are dictated by the same quantity, $v_{\chi}$, the scale of symmetry breaking of the 3-3-1 symmetry. Indeed one can find that $M_{W^{\prime}} \sim 0.32 v_{\chi}$ and $M_{Z^{\prime}} \sim$ $0.4 v_{\chi}$. Limits from dilepton searches at the LHC at $14 \mathrm{TeV}$ with $\sim 23 \mathrm{fb}^{-1}$ exclude $M_{Z^{\prime}}<4 \mathrm{TeV}$ (see Figure 9 inside [49]). With a projection with $100 \mathrm{fb}^{-1}$ of data, extrapolating the luminosity effect, one would exclude $M_{Z^{\prime}}<4.7 \mathrm{TeV}$. These two limits translate into $M_{W^{\prime}}>3.2 \mathrm{TeV}$ and $M_{W^{\prime}}>3.7 \mathrm{TeV}$, respectively.

Therefore, we can conclude that the neutrinoless double beta decay offers a complementary probe to collider physics and, depending on choices for $v_{\sigma 1}$, it can offer the most restrictive bound on this gauge boson mass. We emphasize that the mixing angle $\theta$ is quite small since it is dictated by $M_{W W^{\prime}}^{2} / M_{W^{\prime}}^{2}$, rendering our conclusions robust.

\section{Validity of the Minimal 3-3-1 Model}

It has been shown that the $3-3-1 \mathrm{M}$ is valid up to energies of $5.7 \mathrm{TeV}$ or so due the presence of a Landau pole $[12,50]$. The collider bounds on the $Z^{\prime}$ and $W^{\prime}$ mentioned above already pose a strong tension on the model since these mass limits translate into $v_{\chi} \approx 10 \mathrm{TeV}$ and $v_{\chi} \approx 12 \mathrm{TeV}$. With the addition of new exotic fermions that can contribute to the renormalization group equations, this tension can be alleviated. As can be seen in Figure 5, for $m_{\beta \beta} \sim 0.1 \mathrm{eV}$ and $v_{\sigma 1} \sim 10^{-6} \mathrm{GeV}$, the lower mass bound on $W^{\prime}$ lies around 1.6 TeV, which implies that $v_{\chi}>3.6 \mathrm{TeV}$.

This would constitute a strong but valid claim, under the assumptions made. Neutrinoless double beta decay under certain assumptions already excludes the minimal 3-3-1 model, imposing that the scale of symmetry breaking of the model should lie beyond its validity (for previous bounds on this model see [51-59]).

These findings are valid for $m_{\beta \beta} \sim 0.1 \mathrm{eV}$, but if we consider the nEXO sensitivity which is expected to reach $m_{\beta \beta} \sim 0.01 \mathrm{eV}$, the impact that our study brings to the minimal 3-3-1 model is even more profound. Even for $v_{\sigma 1} \sim$ $10^{-6} \mathrm{GeV}$, we would already impose $v_{\chi} \geq 6 \mathrm{TeV}$.

In summary, neutrinoless double beta decay offers an orthogonal probe to the minimal 3-3-1 model. If the vevs of the fields in the scalar sextet are sufficiently small, neutrinoless double beta decay does not favor the original version of the minimal 3-3-1 model.

\section{Conclusions}

We discussed neutrinoless double beta decay in the context of the minimal 3-3-1 model. It features a minimal fermion content, arguably rendering it more predictive in comparison to other models based on this 3-3-1 gauge group. In the past decades, we have observed a significant improvement in the bound of the neutrinoless double beta decay. We exploit this fact to obtain a lower mass bound on the $W^{\prime}$ boson that arises due to the enlarged gauge group. Our limits are based mostly on the charged current and the vev of a scalar field that generates neutrino masses and induces the $W-W^{\prime}$ mixing. This mixing leads to a sizable contribution to neutrinoless double beta decay.

We obtained a fully analytic expression that represents a lower mass bound on the $W^{\prime}$ mass. Depending on the vev adopted for this scalar field $\left(\sigma_{1}\right)$, neutrinoless double beta decay might offer the most restrictive limit on the $W^{\prime}$ surpassing those of collider probes. Moreover, since the $W^{\prime}$ mass is directly connected to the scale of symmetry breaking 
of the model, a lower mass bound on the gauge boson represents also a lower bound on the scale of symmetry breaking. In particular, if $v_{\sigma 1}>0.1 \mathrm{GeV}$, current limits on the half-life of the neutrinoless double beta decay strongly disfavored out the minimal 3-3-1 model.

\section{Conflicts of Interest}

The authors declare that there are no conflicts of interest regarding the publication of this paper.

\section{Acknowledgments}

This work was supported by Coordenação de Aperfeiçoamento de Pessoal de Nível Superior (Capes-PDSE88881.135139/2016-01). The authors are also grateful to Farinaldo Queiroz and Diego Cogollo for discussions and suggestions.

\section{References}

[1] C. Patrignani et al., "Review of Particle Physics," Chinese Physics C, vol. 40, no. 10, Article ID 100001, 2016.

[2] W. Rodejohann, "Neutrino-less double beta decay and particle physics," International Journal of Modern Physics E, vol. 20, no. 9, pp. 1833-1930, 2011.

[3] H. Päs and W. Rodejohann, "Neutrinoless double beta decay," New Journal of Physics, vol. 17, no. 11, Article ID 115010, 2015.

[4] M. Singer, J. W. F. Valle, and J. Schechter, "Canonical neutralcurrent predictions from the weak-electromagnetic gauge group SU(3) × U(1)," Physical Review D: Particles, Fields, Gravitation and Cosmology, vol. 22, no. 3, pp. 738-743, 1980.

[5] F. Pisano and V. Pleitez, "SU(3) $\otimes U(1)$ model for electroweak interactions," Physical Review D: Particles, Fields, Gravitation and Cosmology, vol. 46, no. 1, pp. 410-417, 1992.

[6] R. Foot, O. F. Hernández, F. Pisano, and V. Pleitez, "Lepton masses in an $S U(3)_{L} \times U(1)_{N}$ gauge model," Physical Review D: Particles, Fields, Gravitation and Cosmology, vol. 47, no. 9, pp. 4158-4161, 1993.

[7] P. Frampton, "Chiral dilepton model and the flavor question," Physical Review Letters, vol. 69, no. 20, pp. 2889-2891, 1992.

[8] R. Foot, H. N. Long, and T. A. Tran, " $S U(3) L \otimes U(1) N$ and $S U(4) L \otimes U(1) N$ gauge models with right-handed neutrinos," Physical Review D: Particles, Fields, Gravitation and Cosmology, vol. 50, no. 1, pp. R34-R38, 1994.

[9] H. N. Long, “The 331 model with right handed neutrinos," Physical Review D: Particles, Fields, Gravitation and Cosmology, vol. 53, pp. 437-445, 1996, https://arxiv.org/abs/hep-ph/9504274.

[10] A. Alves, E. Ramirez Barreto, A. G. Dias, C. A. de S. Pires, F. S. Queiroz, and P. S. Rodrigues da Silva, "Explaining the Higgs decays at the LHC with an extended electroweak model," The European Physical Journal C, vol. 73, no. 2, article no. 2288, pp. 1-9, 2013.

[11] W. Caetano, C. A. de S. Pires, P. S. Rodrigues da Silva, D. Cogollo, and F. S. Queiroz, "Explaining ATLAS and CMS results within the reduced minimal 3-3-1 model," The European Physical Journal C, vol. 73, no. 10, article no. 2607, pp. 1-10, 2013.

[12] A. G. Dias, R. Martinez, and V. Pleitez, "Concerning the Landau pole in 3-3-1 models," The European Physical Journal C, vol. 39, no. 1, pp. 101-107, 2005.
[13] A. G. Dias, "Evading the few TeV perturbative limit in 3-3-1 models," Physical Review D: Particles, Fields, Gravitation and Cosmology, vol. 71, no. 1, Article ID 015009, 2005.

[14] C. A. de S. Pires, F. Queiroz, and P. S. Rodrigues da Silva, "A minimal 3-3-1 model with naturally sub-eV neutrinos," Physical Review D: Particles, Fields, Gravitation and Cosmology, vol. 82, no. 6, Article ID 065018, 2010, https://arxiv.org/abs/1003.1270v2.

[15] W. Caetano, D. Cogollo, C. A. D. S. Pires, and P. S. R. Da Silva, "Combining type I and type II seesaw mechanisms in the minimal 3-3-1 model," Physical Review D: Particles, Fields, Gravitation and Cosmology, vol. 86, no. 5, Article ID 055021, 2012.

[16] D. Cogollo, H. Diniz, and C. A. de S. Pires, "keV right-handed neutrinos from type II seesaw mechanism in a 3-3-1 model," Physics Letters B, vol. 677, no. 5, pp. 338-342, 2009.

[17] S. Profumo and F. S. Queiroz, "Constraining the $Z^{\prime}$ mass in 331 models using direct dark matter detection," The European Physical Journal C, vol. 74, no. 7, article no. 2960, pp. 1-12, 2014.

[18] A. Alves, G. Arcadi, P. V. Dong, L. Duarte, F. S. Queiroz, and J. W. F. Valle, "R-parity as a residual gauge symmetry: probing a theory of cosmological dark matter," Physics Letters B, vol. 772, pp. 825-831, 2017.

[19] D. Cogollo, F. S. Queiroz, and P. Vasconcelos, "Flavor changing neutral current processes in a reduced minimal scalar sector," Modern Physics Letters A, vol. 29, no. 32, Article ID 1450173, 2014.

[20] F. S. Queiroz, C. Siqueira, and J. W. F. Valle, "Constraining flavor changing interactions from LHC Run-2 dilepton bounds with vector mediators," Physics Letters B, vol. 763, pp. 269-274, 2016.

[21] A. Alves, S. Profumo, F. S. Queiroz, and W. Shepherd, "Effective field theory approach to the Galactic Center gamma-ray excess," Physical Review D: Particles, Fields, Gravitation and Cosmology, vol. 90, no. 11, Article ID 115003, 2014.

[22] M. D. Campos, F. S. Queiroz, C. E. Yaguna, and C. Weniger, "Search for right-handed neutrinos from dark matter annihilation with gamma-rays," Journal of Cosmology and Astroparticle Physics, vol. 2017, no. 7, article no. 016, 2017.

[23] A. Alves, A. Berlin, S. Profumo, and F. S. Queiroz, "Diracfermionic dark matter in $U(1)_{X}$ models," Journal of High Energy Physics, vol. 2015, article 76, pp. 1-33, 2015.

[24] A. Alves, A. Berlin, S. Profumo, and F. S. Queiroz, "Dark Matter Complementarity and the $Z$ ' Portal," Physical Review D, vol. 92, no. 8, Article ID 083004, 2015.

[25] A. Doff and C. Siqueira, "Composite Higgs models, Technicolor and the muon anomalous magnetic moment," Physics Letters B, vol. 754, pp. 294-301, 2016.

[26] J. G. Ferreira, C. A. de S. Pires, J. G. Rodrigues, and P. S. Rodrigues da Silva, "Embedding cosmological inflation, axion dark matter and seesaw mechanism in a 3-3-1 gauge model," Physics Letters B, vol. 771, pp. 199-205, 2017.

[27] O. Rodríguez, R. H. Benavides, W. A. Ponce, and E. Rojas, "Flipped versions of the universal 3-3-1 and the left-right symmetric models in [SU (3)] 3: A comprehensive approach," Physical Review D: Particles, Fields, Gravitation and Cosmology, vol. 95, no. 1, Article ID 014009, 2017.

[28] C. A. de S. Pires, P. S. R. Da Silva, A. C. O. Santos, and C. Siqueira, "Higgs mass and right-handed sneutrino WIMP in a supersymmetric 3-3-1 model," Physical Review D: Particles, Fields, Gravitation and Cosmology, vol. 94, no. 5, Article ID 055014, 2016.

[29] J. S. Borges and R. O. Ramos, "Symmetry breaking patterns of the 3-3-1 model at finite temperature," The European Physical Journal C, vol. 76, no. 6, 2016. 
[30] P. V. Dong, D. Q. Phong, D. V. Soa, and N. C. Thao, The economical 3-3-1 model revisited,

[31] J. G. Ferreira, C. A. de S. Pires, P. S. Rodrigues da Silva, and C. Siqueira, "On the Higgs-like boson in the minimal supersymmetric 3-3-1 model," The European Physical Journal C, vol. 78 , no. $225,2018$.

[32] J. C. Montero, C. A. de S. Pires, and V. Pleitez, "Neutrino masses through the seesaw mechanism in 3-3-1 models," Physical Review D: Particles, Fields, Gravitation and Cosmology, vol. 65, no. 9, Article ID 095001, 2002.

[33] J. C. Montero, C. A. de S. Pires, and V. Pleitez, "Lepton masses from a TeV scale in a 3-3-1 model," Physical Review D: Particles, Fields, Gravitation and Cosmology, vol. 66, no. 11, Article ID 113003, 2002.

[34] R. N. Mohapatra, A. Pérez-Lorenzana, and C. A. de Sousa Pires, "Type II seesaw and a gauge model for the bimaximal mixing explanation of neutrino puzzles," Physics Letters B, vol. 474, no. 3-4, pp. 355-360, 2000.

[35] P. Gu, H. Zhang, and S. Zhou, "Minimal type II seesaw model," Physical Review D: Particles, Fields, Gravitation and Cosmology, vol. 74, no. 7, Article ID 076002, 2006.

[36] E. K. Akhmedov and M. Frigerio, "Interplay of type I and type II seesaw contributions to neutrino mass," Journal of High Energy Physics, vol. 2007, no. 01, 2007.

[37] M. Reig, J. W. F. Valle, and C. A. Vaquera-Araujo, "Realistic $\mathrm{SU}(3)_{c} \otimes \mathrm{SU}(3)_{L} \otimes \mathrm{U}(1)_{X}$ model with a type II Dirac neutrino seesaw mechanism," Physical Review D: Particles, Fields, Gravitation and Cosmology, vol. 94, no. 3, Article ID 033012, 2016.

[38] J. C. Montero, C. A. de S. Pires, and V. Pleitez, "Spontaneous breaking of a global symmetry in a 3-3-1 model," Physical Review D: Particles, Fields, Gravitation and Cosmology, vol. 60, Article ID 115003, 1999.

[39] J. C. Montero, C. A. de Sousa Pires, and V. Pleitez, "Comment on 'Majoron emitting neutrinoless double beta decay in the electroweak chiral gauge extensions,' Physical Review D: Particles, Fields, Gravitation and Cosmology, vol. 60, no. 9, Article ID $098701,1999$.

[40] J. C. Montero, C. A. de Sousa Pires, and V. Pleitez, "Neutrinoless double beta decay with and without Majoron-like boson emission in a 3-3-1 model," Physical Review D: Particles, Fields, Gravitation and Cosmology, vol. 64, no. 9, Article ID 096001, 2001.

[41] J. C. Montero, C. A. de S. Pires, and V. Pleitez, "Seesaw tau lepton mass and calculable neutrino masses in a 3-3-1 model," Physical Review D: Particles, Fields, Gravitation and Cosmology, vol. 65, no. 9, Article ID 093017, 2002.

[42] P. V. Dong and D. T. Si, "Discriminating the minimal 3-3-1 models," Physical Review D: Particles, Fields, Gravitation and Cosmology, vol. 90, no. 11, Article ID 117703, 2014.

[43] F. S. Queiroz, K. Sinha, and A. Strumia, "Leptoquarks, dark matter, and anomalous LHC events," Physical Review D: Particles, Fields, Gravitation and Cosmology, vol. 91, no. 3, Article ID 035006, 2015.

[44] B. Allanach, A. Alves, F. S. Queiroz, K. Sinha, and A. Strumia, "Interpreting the CMS $\ell^{+} \ell^{-} j j+$ missing transverse energy excess with a leptoquark model," Physical Review D: Particles, Fields, Gravitation and Cosmology, vol. 92, no. 5, Article ID 055023, 2015.

[45] S. Dell'Oro, S. Marcocci, M. Viel, and F. Vissani, "Neutrinoless double beta decay: 2015 review," Advances in High Energy Physics, vol. 2016, Article ID 2162659, 37 pages, 2016.

[46] K. Asakura et al., "Results from KamLAND-Zen," Article ID 170003, 1666, 2015, AIP Conf. Proc.
[47] D. V. Soa, P. V. Dong, T. T. Huong, and H. N. Long, “Bilepton contributions to the neutrinoless double beta decay in the economical 3-3-1 model," Journal of Experimental and Theoretical Physics, vol. 108, no. 5, pp. 757-763, 2009.

[48] S. Shelly Sharma, "Reply to "Comment on 'Majoron emitting neutrinoless double beta decay in the electroweak chiral gauge extensions' "," Physical Review D: Particles, Fields, Gravitation and Cosmology, vol. 60, no. 9, Article ID 098702, 1999.

[49] Y. A. Coutinho, V. S. Guimarães, and A. A. Nepomuceno, "Bounds on Z' from 3-3-1 model at the LHC energies," Physical Review D: Particles, Fields, Gravitation and Cosmology, vol. 87, no. 11, Article ID 115014, 2013.

[50] R. Martínez and F. Ochoa, "The Landau pole and Ź decays in the 331 dilepton model," The European Physical Journal C, vol. 51, no. 3, pp. 701-711, 2007.

[51] R. A. Diaz, R. Martínez, and F. Ochoa, "Scalar sector of the $S U(3)_{c} \otimes S U(3)_{L} \otimes U(1)_{X}$ model," Physical Review D: Particles, Fields, Gravitation and Cosmology, vol. 69, no. 9, Article ID 095009, 2004.

[52] R. A. Diaz, R. Martinez, and F. Ochoa, " $S U(3)_{c} \otimes S U(3)_{L} \otimes U(1)_{X}$ models for $\beta$ arbitrary and families with mirror fermions," Physical Review D: Particles, Fields, Gravitation and Cosmology, vol. 72, no. 3, Article ID 035018, 2005.

[53] G. A. Gonzalez-Sprinberg, R. Martinez, and O. Sampayo, "Bilepton and exotic quark mass limits in 331 models from Z > b anti-b decay," Physical Review D: Particles, Fields, Gravitation and Cosmology, vol. 71, Article ID 115003, 2005.

[54] F. Ochoa and R. Martínez, "Family dependence in $S U(3)_{c} \otimes$ $S U(3)_{L} \otimes U(1)_{X}$ models," Physical Review D: Particles, Fields, Gravitation and Cosmology, vol. 72, no. 3, Article ID 035010, 2005.

[55] A. E. Carcamo Hernandez, R. Martínez, and F. Ochoa, " $Z$ and $Z$ ' decays with and without FCNC in 331 models," Physical Review D: Particles, Fields, Gravitation and Cosmology, vol. 73, no. 3, Article ID 035007, 2006.

[56] A. G. Dias, A. Doff, C. A. De S. Pires, and P. S. Rodrigues Da Silva, "Neutrino decay and neutrinoless double beta decay in a 3-3-1 model," Physical Review D: Particles, Fields, Gravitation and Cosmology, vol. 72, no. 3, Article ID 035006, pp. 1-8, 2005.

[57] J. M. Cabarcas, D. Gómez Dumm, and R. Martinez, "Phenomenological aspects of the exotic T quark in 331 models," The European Physical Journal C, vol. 58, no. 4, pp. 569-578, 2008.

[58] R. Martinez and F. Ochoa, "Heavy quark signals from radiative corrections to the Z-prime boson decay in 3-3-1 models," Physical Review D: Particles, Fields, Gravitation and Cosmology, vol. 80, Article ID 075020, 2009.

[59] J. M. Cabarcas, D. G. Dumm, and R. Martinez, "Flavorchanging neutral currents in 331 models," Journal of Physics G: Nuclear and Particle Physics, vol. 37, no. 4, Article ID 045001, 2010. 

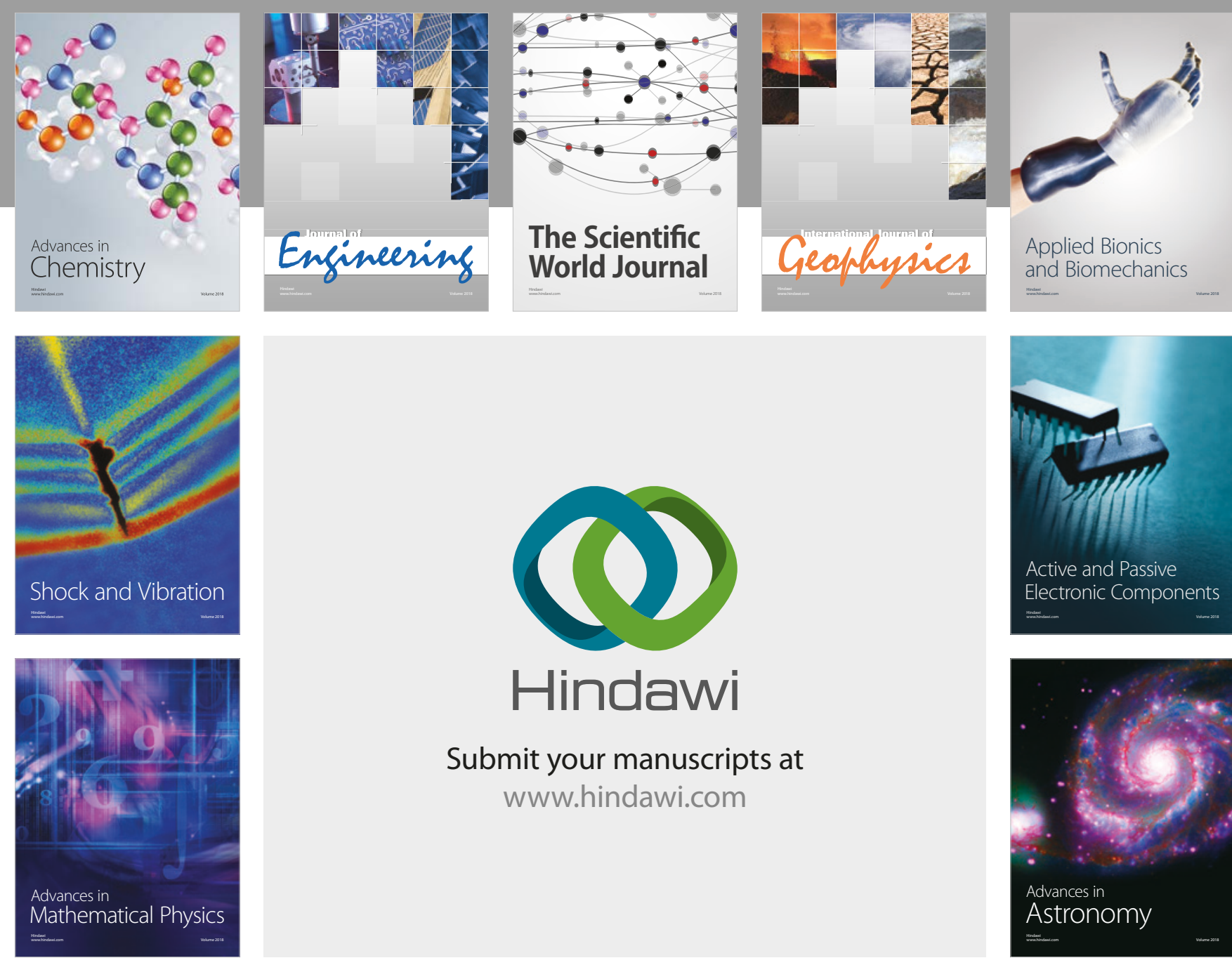

Submit your manuscripts at

www.hindawi.com

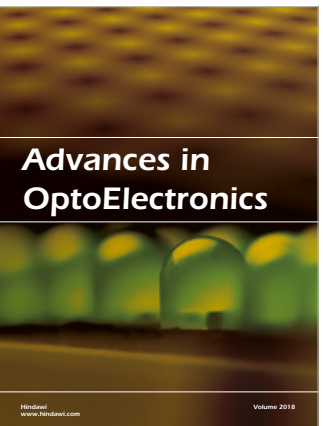

\section{Rotcting Machinery}
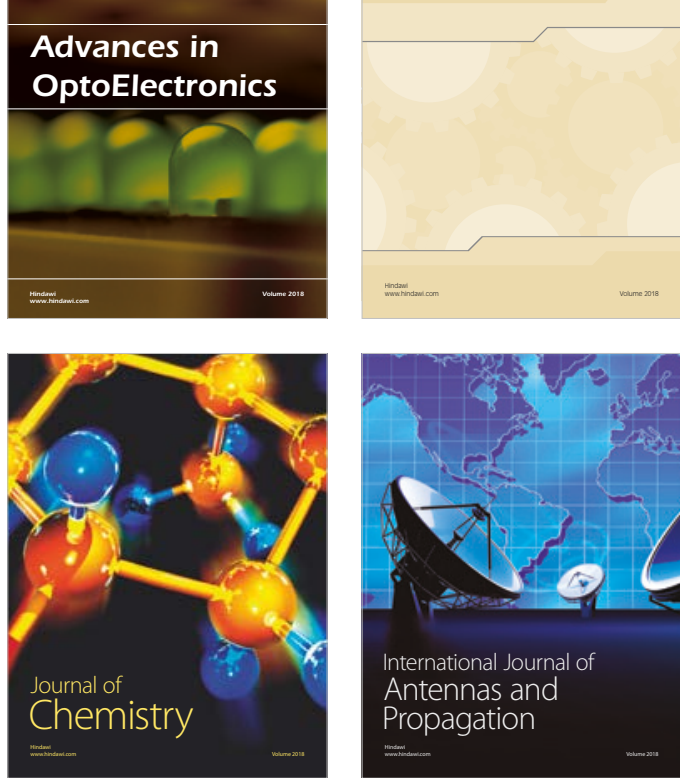

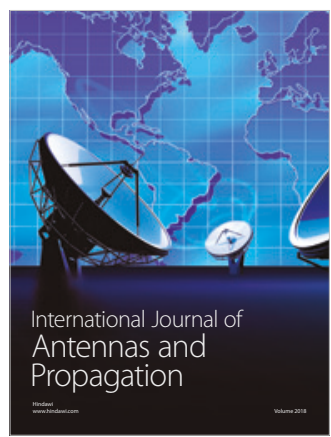

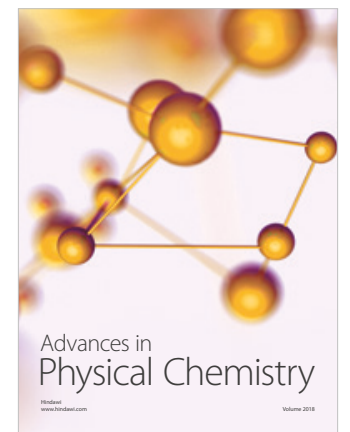

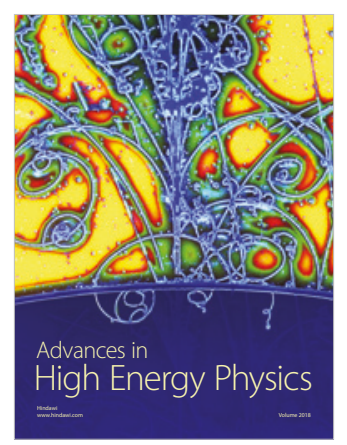

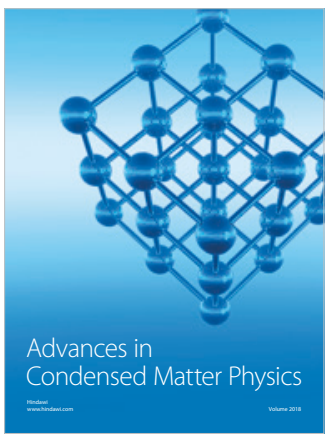

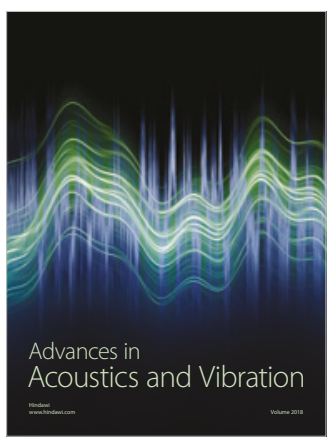

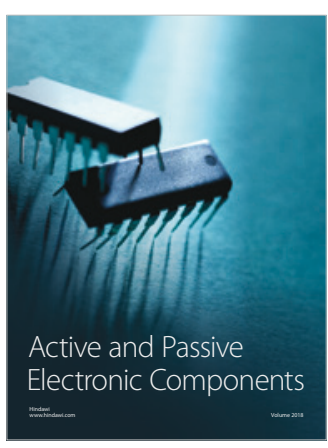
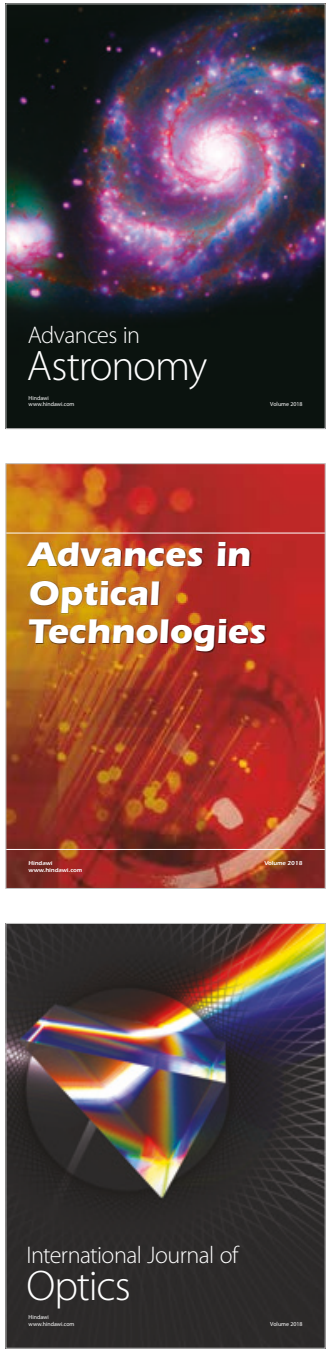